\title{
Manufacturing 4.0: Discussion on Application in the Extractive Industry of Essential Oils
}

\author{
Carlos Alberto Tosta Machado ${ }^{1 *}$; Matheus Antonio Nogueira de Andrade ${ }^{2}$; Herman Augusto Lepikson ${ }^{3}$ \\ ${ }^{I}$ SENAI-CIMATEC, GETEC; ${ }^{2}$ Federal University of Bahia; ${ }^{3}$ SENAI-CIMATEC, IIA;Salvador, Bahia, Brazil
}

\begin{abstract}
Yield uncertainties characterize the industrial extraction of essential oils. The variable plant contents, undetected and uncorrected operational issues such as production in small batches, with a significant variation in the process cycles, productivity, and quality, compromise process performance, and the value of the finished product. The technological advances of industry 4.0 provide an opportunity to achieve economic, environmental, and productivity gains, even with the wide variety of products manufactured. This study aimed at improving the performance of such processes using the pillars of industry 4.0 as a method, highlighting the techniques of data acquisition and processing for intelligent analysis, based on automated and systemic learning, generating improvements for the overall performance of the operation as well as enhancing good manufacturing practices (process safety, quality, repeatability and traceability) and supply chain management. Our findings showed the adherence of 4.0 vision to this industry.
\end{abstract}

Keywords: Manufacturing 4.0. Essential Oils. Supply Management.

Essential oils are natural products extracted from plant parts (flowers, bark, stem, leaves, roots, fruits, and seeds) with essential applications in the food, beverage, cosmetics and pharmaceutical industries, and as alternative treatments for aromatherapy (baths, inhalation, massage and topical applications), as well as biological fungicides, pesticides and antioxidants [1]. An essential oil consists of a range of components, from the most volatile to the heaviest.

According to Research and Markets, March 2019, the global market for essential oils is expected to reach $\$ 16.9$ billion by 2026 , growing at an average rate of $10.8 \%$ during this period [2].

Besides the diversity of species from which essential oils are extracted, seasonal variations also impact the products obtained, which may vary significantly in composition and quantities, with evident impacts on agronomic and economic aspects [3]. Therefore, the extractive technology appears like an essential asset since not only the

Received on 28 August 2019; revised 29 September 2019. Address for correspondence: Dr. Carlos Alberto Tosta Machado. Avenida Orlando Gomes, N. 1845, Piatã. Zip Code: 41650-010. Salvador, Bahia, Brazil. E-mail: carlos.tosta@ uol.com.br. This study was selected from the V International Symposium of Innovation and Technology - SIINTEC (October 2019).

J Bioeng. Biotech. Appl. Health 2019;2(3):86-91.

(C) 2019 by SENAI CIMATEC. All rights reserved. methods and equipment used have importance over the obtained products, but also the embedded control technology and the self-configuration of the production facilities, adapting the process to the specific content in the binomial plant and period of its harvest.

The industry segment of essential oils presents new opportunities for technological upgrades [4]. The introduction of manufacturing 4.0 pillars aimed in this study the improvement of the overall performance of the essential oil extraction industry.

The multidisciplinary aspect of this industrial sector leads to the need to integrate the knowledge of Chemistry, Biology, and Technology (Figure 1) [5].

The chemical aspects are related to the integrity of the volatile products obtained; the biological aspects refer to the behavior of the plant in the extractive process, which enable understanding where the essential oils were deposited in the cellular structure; and the technology ensures the previous aspects as well as productivity and process safety.

Among the essential extractive methods, steam distillation, subcritical carbon dioxide application, cold pressing, and solvent application, concentrate the majority of worldwide production. This manuscript focused on steam distillation, providing insight into the technological possibilities in 
Figure 1. Multidisciplinary aspect of performance in the essential oil industry.

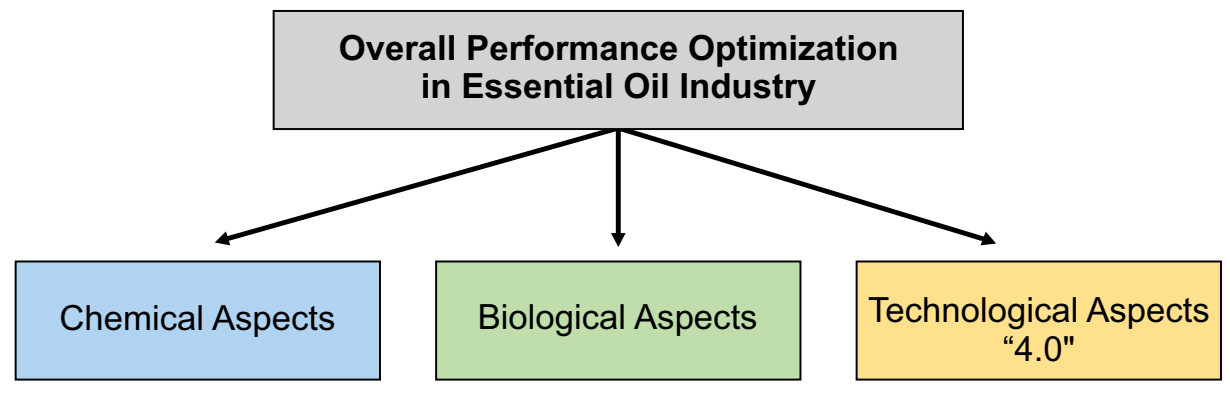

quality, productivity, and sustainability, that providing opportunities for improvements in the business operating margins.

The steam crosses the plant material inside the processing vessel and the product is, then, condensed. The density difference separates this water-oil mix. The supernatant is the essential oil, and the rest of the condensate is called hydrolate, which also has commercial value (Figure 2).

The application of water/steam as a solvent brings environmental benefits in the extraction and purification processes. Nonpolar solvents (hydrocarbons) have significant negative environmental impact and require careful handling during production and subsequent separation [5].
This manuscript creates a 4.0 perspective such as connectivity and real-time process' visibility, which allows identification and correction of deviations and failures, where the control system acquires parameters and optimizes procedures, incorporating them into standard operations [6], autonomously and, in an adaptive way, generating a database for traceability and verification of process repeatability.

The backbones of industry 4.0 comprise a set of technological possibilities that aim to integrate the operational areas of companies. Such composition creates a Cyber-Physical System (CPS), in which the physical and computational components will be deeply braided [10].

Figure 2. Schematic representation of water steam distillation.

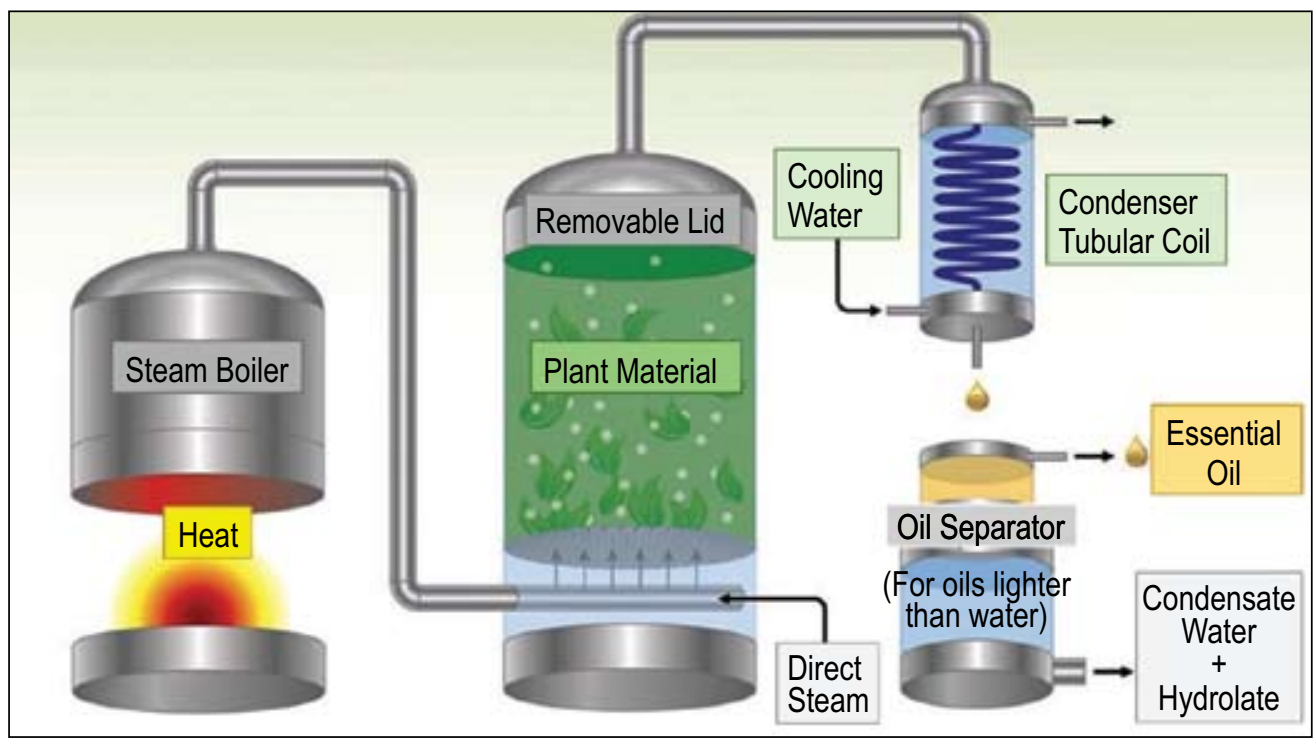

Source: www.doterra.com 
This higher technological level has been demonstrated in this article, resulting in additional enhancements for several operational and business areas. It is a consistent competitive opportunity in a growing and increasingly demanding market [7]. The opportunities' areas brought by the authors address the 4.0 mainstays considered appropriate, as a technological situation in the reading of this business segment.

\section{Methods}

The method tried to understand the product (essential oils) and the current state of the industry in this area through visits, and conference calls with national and international researchers - Prof. Ph.D. Farid Chemat - Avignon University, France; Prof. Dr. Maria Angela Meireles - UNICAMP and Prof. Dr. Martins Dias de Cerqueira - UFBA) and visiting companies in this field (LINAX, DIERBERGER) - from February / 2019 to July / 2019.

Systematic bibliographic research completed the formation of a proposal for 4.0 industry's improvement.

Also, good manufacturing practices (process safety, quality, repeatability, and traceability) and supply chain management were discussed in the supplier's and customer's interfaces, as the industrial process is not dissociated from these significant links.

This paper focused on industrial processes of extracting essential oils by hydrodistillation with a proposed operational and control model, applying the concepts of manufacturing 4.0: data acquisition systems through the intensive intelligent application of sensors - analytical processes that generate learning automatic and systemic with advantages for the branch concerned.

\section{Results and Discussion}

\section{Describing the Opportunities}

One point has become unanimous among all the researchers and companies: empiric approaches base the essential oil production process, using facilities still not modernized, applying the same set of operating practices to all types of products, without the necessary differentiation $[4,5]$. Such procedures bring yield uncertainties and unwanted variations in quality, and others process.

So, it is necessary to improve:

- Material Yield Improvement: Plants from the same crop (raw material) generate different yields of essential oil.

- Process Time: Lack of repeatability even with similar materials. Dependence on empiricism-based interventions.

- Variable Purity and Quality: Turbidity, coloration, and - especially - quantification of all essential oil components.

- Variable energy consumption from batch to batch.

- Variation in solvent consumption (water steam).

- Non-automatic cleaning procedures bringing uncertainties to the next batch, facility readiness and contamination risks.

- Degradation of essential oil components by overexposure to overheated points, reflecting lack of control.

- Effluent generation beyond the minimum possible.

- Impacts on product operating margins as a consequence of all previous items.

\section{Good Manufacturing Practice Aspects}

The production of essential oils will be subject to legal requirements based on good manufacturing practices, according to ANVISA RDC 17. (Collegiate Board Resolution of October 16, 2010), as they are used as raw materials for cosmetics and pharmaceuticals. Physical facilities should be following this regulatory guideline, also meeting repeatability and traceability requirements.

\section{Supply Chain Aspects}

The reliability of supply - timeliness, quality, and product integrity (meeting physicochemical specifications) - clearly brings recognition from 
customers, which goes far beyond the negotiated price: the reliability in service (safety, quality, and timeliness) of companies in the cosmetics and pharmaceutical industries. Hence the importance of robust industrial processes that achieve their global operational objectives for the first time. An eventual correction, in view 4.0, will be assimilated by the process (machine learning), incorporating the improvements for future batches.

\section{Discussion of a Method for Overall Performance Improvement}

We proposed a set of 4.0 recommendations in this manuscript, whose applicability is appropriate in the items: Describing the Opportunities; Good Manufacturing Practice Aspects; and Supply Chain Aspects.

\section{Yield Variations}

Variations in material balance, from batch to batch (material yield or yield), occur primarily due to lack of effective control of vapor flow through the plant bed. Except for differences in particle size of the ground plants, variations in the amount of product obtained come from either the quality of the plant material or the production process. If we assumed that the materials are from the same harvest, then the process would be the source of variation considered.

Numerous articles show mathematical models (References). However, the simplifications lack representativeness, based on the reality of the manufacture (for instance, minimal, nonrepresentative quantities), such as the repeated simplifying hypothesis of an isotropic plant distribution (with homogeneous and compacted porosity without any variations), the hypothesis of smooth and perfectly radial flow of steam (regardless of preferred paths, called channeling) $[3,8,9]$, and other issues.

The proposed action is a digital twin (Figure 3 ), which receives signals from field sensors, in parallel with the actual process, and adapts itself to repeat process variables reliably: yield, time, and energy consumption.

The question on how to detect channeling is crucial, i.e., the shortcut of the steam stream through the raw material. This phenomenon has

Figure 3. Functions of a digital twin.

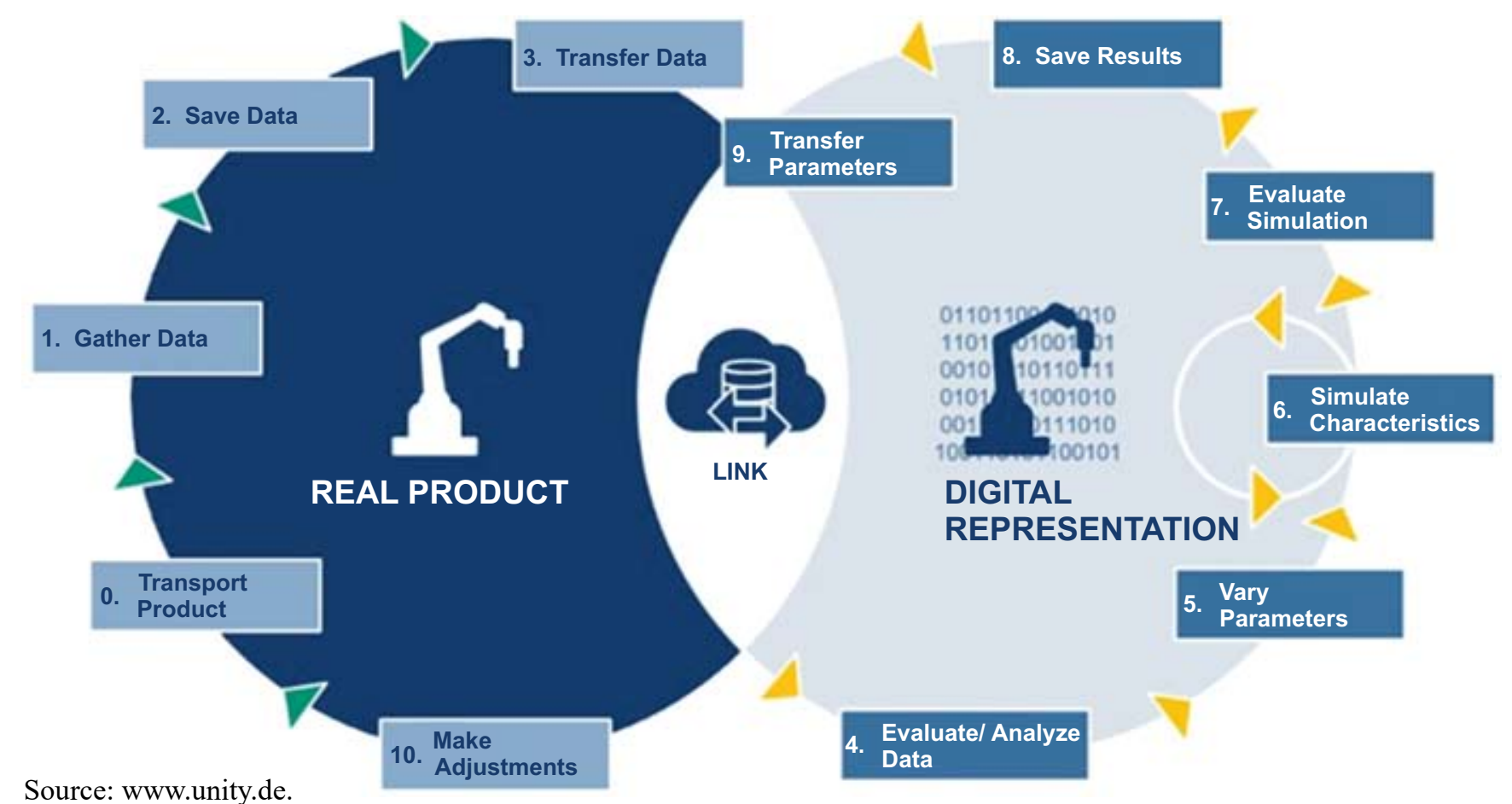


two main negative impacts. First of all, some of the raw material is unreached by the steam-generating yield losses; and the overexposure of some spots partially degrade the product. The best approach to detect this uncertainty is by applying external temperature detection (infrared detection or those called "skin point" sensors) in the extractor vessel. Several temperature transmitters distributed within the diameter and depth would be an expensive alternative. The suitable instrumentation and a reliable digital twin lead to the autonomous learning process (machine learning) and early detection of any deviations.

\section{Process Time}

It is not acceptable that plants from the same crop generate different process times, nor that different plants have the same treatment indiscriminately. These facts reflect a lack of monitoring and control of the process variables: steam distribution, flow, and pressure, raw material degree of compaction within the vessel. Thus, the process should be repetitive, without requiring intermediate inspections, with process interruptions by the operator.

Real-time data (measurements of condensed quantities, for instance) should follow a pattern (set in previous standard batches) to the digital twin. This database, physical or in the cloud, is subject to analysis and applied to dynamically and continuously improve the overall performance.

\section{Purity and Quality Variations}

Density, viscosity, and turbidity should be monitored in real-time. The trend of these indicators creates databank for the digital twin, providing automatic learning and consistency.

\section{Variations in Energy and Solvent Consumption (Water)}

The variations in energy and solvent consumption (water) directly impact product cost and yield, depending on how much they go up or down. The steam consumption curve must be monitored to ensure yield and quality. Energy losses, in addition to the environmental impact, reduce the operating profit margin, decreasing the company's competitiveness.

\section{Cleaning Processes}

Cleanliness becomes a significant risk-factor when the critical process is applied. Crosscontamination both from previous batches and due to unwashed cleaning products may lead to unwanted losses, failing the customer service agreement (delay) and financially. The manual cleaning process, widespread in this industry, indeed introduces such risks. The introduction of an automatic cleaning system using spray balls, with pressure, temperature and flow controls as well as the additive dosing, washing, rinsing and drying with proper monitoring, not only by the time factor but through indicators of the output fluids $(\mathrm{pH}$, conductivity, among other possible examples). CIP (cleaning in place) systems are widely used in the pharmaceutical and cosmetics industry. The database allows analysis and optimization when virtually controlled, in real-time, by the digital twin. For instance, if the final rinse water parameters are related to the wash temperature, the control system may suggest feasibility tests for shortening times and using smaller amounts of water, cleaning products, and energy.

\section{Degradation of Essential Oils}

Product degradation or denaturation occurs due to overexposure to high temperature, oxidation, light exposure, contact with products left from previous batches, or improper cleaning process. The use of an inert atmosphere and reduction of light exposure (closed processes) can solve the mentioned factors.

\section{Effluent Generation}

The environmental consequence of uncontrolled processes is direct: solvent and energy usage beyond the optimal. All devices and methods aforementioned will have a positive effect. The optimization of material usage (raw material consumption) will generate less processed material (called bagasse) and less planted areas. 
Operating Margins Impact

Higher material yields overwhelming boost business financial results in an industry characterized by insufficient material yields (for instance, $100 \mathrm{~mL}$ of product from 3 tons of plants). These products are refined, but increments of a few percentage points are welcome. Process time gain directly increases production capacity, eliminating downtime, waiting, and other issues, improving market response speed, and somehow delaying capacity investments.

\section{Conclusion}

The extraction process of essential oils, when improved by the application of industry 4.0 concepts, can deliver significant advantages from material and energy yields to positive impacts on environmental and financial results. It is not a case of "one size fits all"; therefore, each company will plan investments in the specific 4.0 areas, depending on the technological, economicfinancial, compliance and expected benefits returns. It is, therefore, a matter of an oriented choice, developing the maturity to select projects to comprise the technological future of the business. Enhanced operational and financial results will provide new days to the organization, allowing the creation of a relentless behavior of pursuing new technical and operational horizons of excellence. Nevertheless, this is a subject for further studies.

\section{References}

1. Tisserand, R., Young, R. Essential oil safety-e-book: A guide for health care professionals. Elsevier Health Sciences. 2013.

2. https://www.researchandmarkets.com/reports/4769350/ global-essential-oil-market-analysis-2019\#pos-1.

3. Xavier, V. B., Vargas, R.M.F., Cassel, E., Lucas, A.M., Santos, M.A., Mondin, C.A., Sartor, T. Mathematical modeling for extraction of essential oil from Baccharis spp. by steam distillation. Industrial Crops and Products. 2011;33(3):599-604.

4. Chemat, F., Abert-Vian, M., Fabiano-Tixier, A. S., Strube, J., Uhlenbrock, L., Gunjevic, V., Cravotto, G. Green extraction of natural products. Origins, current status, and future challenges. TrAC Trends in Analytical Chemistry. 2019.

5. Chemat, F., Strube, J. (Eds.) Green extraction of natural products: theory and practice. John Wiley \& Sons. 2015.

6. Lee, J., Bagheri, B., Kao, H.A. A cyber-physical systems architecture for industry 4.0-based manufacturing systems. Manufacturing Letters. 2015;3:18-23.

7. Squire, R., Song, H. Cyber-physical systems opportunities in the chemical industry: A security and emergency management example. Process Safety Progress. 2014;33(4):329-332.

8. Cassel, E., Vargas, R.M.F., Martinez, N., Lorenzo, D., Dellacassa, E. Steam distillation modeling for essential oil extraction process. Industrial Crops and Products. 2009;29(1):171-176.

9. Sartor, R.B., Secchi, A.R., Soares, R.D.P., Cassel, E. Dynamic simulation of rosemary essential oil extraction in an industrial steam distillation unit. Industrial \& Engineering Chemistry Research. 2011;50(7):3955-3959.

10. Xu, L.D., Xu, E.L., Li, L. Industry 4.0: state of the art and future trends. International Journal of Production Research. 2018;56(8). 